\title{
GEOLOGIA Y ASPECTOS ESTRUCTURALES DEL BORDE OROGÉNICO EN EL EXTREMO SUR DEL CINTURON DON FELICIANO
}

\author{
PEDRO OYHANTÇABAL*,ROSSANA MUZIO*,** e SERGIO DE SOUZA*
}

\begin{abstract}
GEOLOGY AND STRUCTURAL ASPECTS OF THE OROGENIC LIMITIN THE SOUTHERN EXTREME OF THE DOM FELICIANO BELT. An approach, to the structural and geológical evolution of the Southern orogenic Brasiliano limit in Uruguay is presented. As a consequence of sinistral shearing during the Late Brasiliano, the dextral wrench fault (Sarandí del $\mathrm{Yi}-\mathrm{A}^{\mathrm{o}}$ Solís Grande) of probably pre-Brasiliano age, is reactivated with intrusion of syn-transcurrent granites. Post-uplift processes, through alkaline and slightly oversaturated magmatism associated with molassic sediments, are observed.
\end{abstract}

Keywords: Tectonic, magmatism, Brasiliano Cycle, Uruguay.

RESUMEN Se presenta un modelo para la evolución estructural y geológica del borde orogénico sur del Brasiliano, en el Uruguay. Como consecuencia de la cizalla sinestral a fines del Brasiliano, la falla dextral (Sarandí del Yi- $\mathrm{A}^{\circ}$ Solís Grande) de edad probable pre-Brasiliana, es reactivada con intrusión de granitos sintectónicos. Se observan procesos posteriores de levantamiento con magmatismo de alcalino a levemente sobresaturado asociado a sedimentos molásicos.

Palabras claves: Tectónica, magmatismo, Brasiliano, Uruguay.

INTRODUCCIÓN Los avances en el conocimiento de los terrenos brasilianos en el Uruguay han sido recientemente reseñados por Bossi (1989) y Bossi \& Campal (1992).

La evolución del Ciclo Orogénico Brasiliano - Pan Africano ha sido interpretada en términos de Tectónica de Placas desde los trabajos de Perada (1979) y Fragoso César et al (1982). Fragoso César et al $(1987,1990)$, Tommasi et al (1990) y Fernandes et al (1992) establecen nuevas aproximaciones al modelo. En esencia, estos modelos plantean que el Cinturón Orogénico Don Feliciano - Gariep es el resultado del cierre de un océano proterozoico (Adamastor) y la colisión, probablemente oblicua, entre los cratones del Río de la Plata y Kalahari.

De acuerdo con Fragoso César et al (1990), las estructuras mayores se habrían generado en dos estadios: a. cabalgamientos y nappes en la etapa sin-colisional y, b. grandes fajas de cizallamiento sub-verticales, pliegues en padrones de domos y cubetas y fallas inversas en la etapa colisional tardía.

Fernandes et al (1992), basándose en el método de correlación cinemática, plantean la existencia de dos fases cinemáticas: K1 y K2. La primera se caracteriza por cizallamientos sub-horizontales con transporte E-W, en tanto que la segunda expone cizallas sub-horizontales con movimiento N-E del bloque superior y zonas de cizalla con transcurrencia sinestral.

Estas dos grandes etapas son coherentes con lo observado en este trabajo que plantea, en función de lo observado en el Sur del Cinturón Don Feliciano, un modelo para la evolución del área.

El área estudiada se sitúa en el extremo sur del borde orogénico Brasiliano en el Uruguay, donde este último confluye con la falla Sarandí del Yí - ${ }^{\circ}$ - Solís Grande. La zona comprendida entre estos dos grandes elementos estructurales está representada por una faja milonítica, con intrusiones sintranscurrentes asociadas. Al oeste de la falla afloran terrenos correspondientes al Cratón del Río de la Plata, fundamentalmente milonitas afectadas por la falla. Al este del Borde Orogénico se desarrollan las supracrustales del Grupo Lavalleja. El magmatismo de la formación Sierra de Animas y los sedimentos asociados se instalan en estructuras distensivas de dirección NS, utilizando la discontinuidad cizallada entre las supracrustales y la faja milonítica de Aguas Blancas (Fig. 1).

GEOLOGÍA DEL BASAMENTO Zona Occidental del Ao. Solís Grande Milonitas Graniticas Se caracterizan por porfiroclastos de microclina en una matriz foliada compuesta por microclina, plagioclasa, cuarzo,
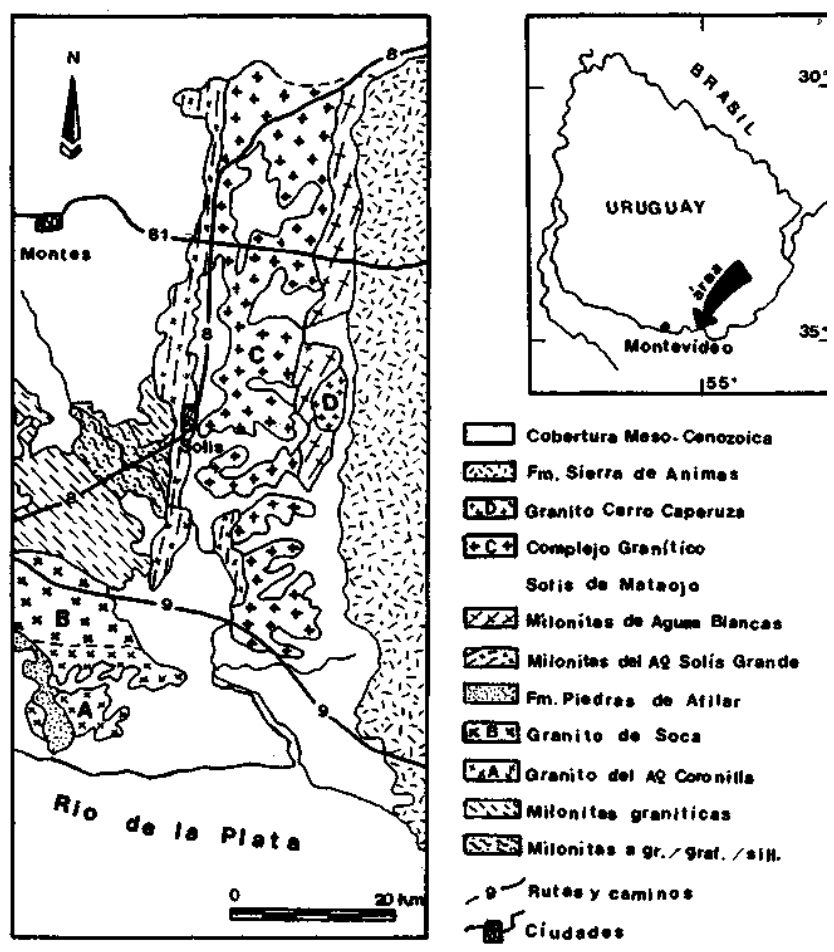

Figura 1 - Mapa de ubicación del área de trabajo Figure 1 - Location of the study area

\footnotetext{
* Facultad de Ciencias, Departamento de Geología, Tristán Narvaja 1674, Casilla 11200, Montevideo, Uruguay

** Post-graduación, Instituto de Geociências e Ciềncias Exatas, Universidade Estadual Paulista, Caixa Postal 178, CEP 13500-970, Rio Claro, Brasil
} 
biotita, muscovita y granate, epidoto y circón como accesorios principales. La deformación que presentan es variable, desarrollándose desde granitos protomiloníticos hasta ultramilonitas y filonitas.

Milonitas a granate, grafito y sillimanita Constituyen una banda de aproximadamente $2 \mathrm{~km}$ de potencia, concordante con la unidad anterior. Son el producto de la deformación de micaesquistos y paraneises bandeados, constituidos por cuarzo, plagioclasa, biotita, muscovita, granate pretectónico, sillimanita, estaurolita, clorita y sericita. Coexiste en estas rocas una paragénesis de grado medio a alto (estaurolita, sillimanita, granate I), junto a otra retrometamórfica (clorita, sericita, granate II).

Granito del A ${ }^{\circ}$ - Coronilla Fue definido por Jones (1956) tratándose de un granito de grano medio a fino, a dos micas, de color gris. Suele observarse una foliación de rumbo N320-N340 y aparece intercalado con esquistos biotíticos con grafito ocasional.

Granito de Soca Esta intrusión fue definida por Jones (1956) como Granodiorita de Tío Diego y redefinida por Bossi et al. (1965) como Granito de Soca. Es un leucogranito alcali-feldespático, hornblendo-biotítico, de grano grueso a muy grueso y color gris verdoso oscuro a rosado. En esta zona está caracterizado por el incremento de la deformación hacia el extremo SE del cuerpo, por efecto de la cizalla dextral Sarandí del Yí - A ${ }^{\mathrm{o}}$ Solís Grande (S.Y.- A.S.G.). El contacto entre este granito y la Faja Cizallada S.Y.- A.S.G. se encuentra cubierto por sedimentos cuaternarios.

Formación Piedras de Afilar Esta unidad fue estudiada inicialmente por Walther (1927), retomada por Jones (1956) y elevada a la categoría de formación por Bossi (1966).

En forma más reciente, Coronel et al (1982) establecieron una secuencia grano decreciente con areniscas gruesas, cuarzosas y a veces cuarzo-feldespática en la base, hacia la parte media areniscas finas, intercaladas con limolitas grises oscuras, micáceas, evolucionando a limolitas calcáreas y calizas en el tope. La secuencia tiene un espesor de aproximadamente $600 \mathrm{~m}$, con una estructura monoclinal de rumbo NW, y buzamiento de 40-50SW. Esta unidad aparece recortada por diabasas espilitizadas. La edad de esta formación no se conoce con exactitud aunque las relaciones estructurales y estratigrafías conocidas la ubican tentativamente en la base del Proterozoico Superior, siguiendo la interpretación de Caorsi \& Goñi (1958), Midot (1984) y Fragoso César et al. (1987).

Zona oriental del $\mathbf{A}^{\mathbf{0}}$ Solís Grande Unidad Milonítica del $\mathrm{A}^{\mathrm{o}}$ Solís Grande Agrupa a rocas de foliación NS-N30. Los protolitos corresponden mayoritariamente a términos supracrustales (micaesquistos con granate y estaurolita, y anfibolitas). No es de descartar que algunas litologías representen estadios de deformación muy avanzada de protolitos graníticos. Las paragénesis presentes en micaesquistos y anfibolitas indican que antes de la milonitización se alcanzaron las condiciones de facies anfibolita.

Complejo Granítico Solís de Mataojo Se trata de un cuerpo muy elongado, de eje mayor NS, con fuerte desarrollo de planaridad concordante y frecuentes enclaves básicos y xenolitos. Está compuesto fundamentalmente por tonalitas hornblendo-biotíticas, granodioritas hornblendobiotíticas a megacristales de microclina y granitos a dos micas. Desde el punto de vista estructural, el cuerpo se caracteriza por presentar un núcleo poco deformado, incrementándose la deformación hacia los bordes oriental y occidental. En el borde este, la deformación se incrementa gradualmente y este granito da lugar en forma parcial a las Milonitas de Aguas Blancas. Un estudio más detallado se encuentra en Oyhantçabal et al. (1993).

Milonitas de Aguas Blancas Conforman una faja de rocas con dirección de foliación dominante NS-N30, situada entre el Complejo Granítico anterior y la formación Sierra de Animas, de la cual se separan a través de un límite de falla. Las litologías dominantes son milonitas hasta ultramilonitas y filonitas. La deformación de esta unidad se incrementa hacia el Este.

Granito del Cerro Caperuza Se trata de una intrusión con escasa a nula deformación, correlacionable con el magmatismo que genera la formación Sierra de Animas. Litológicamente, es un granito rosado, de grano medio, equigranular, con biotita y anfíbol.

Formación Sierra de Animas Es un complejo volcánico-subvolcánico de dirección NS, compuesto por traquitas, sienitas y granitos. Hacia el sur ocurren riolitas ignimbríticas y sedimentos molásicos, englobados y recortados por lavas de esta Formación. La presencia de anfíbol alcalino y augita aegirina como accesorios frecuentes, la ausencia de feldespatoides y la presencia de cuarzo indican un carácter alcalino ligeramente sobresaturado para este magmatismo. Preciozzi et al. (1990) atribuyen los sedimentos arriba mencionados a la formación Piedras de Afilar, pero se considera más conveniente relacionarlos al Grupo Barriga Negra (cf. Preciozzi et al. 1988). En el cerro San Antonio, es posible observar también derrames ignimbríticos asociados a depósitos piroclásticos. Un magmatismo más básico, representado por filones y derrames de lavas vacuolares, es considerado posterior debido a que recorta las litologías sieníticas y traquíticas. Se considera que este complejo volcánicosubvolcánico se ha instalado sobre un rift de dirección NS, hoy relativamente arrasado. Apoyan esta interpretación los sedimentos del Grupo Barriga Negra encontrados en el sur (Playa Verde, Playa Hermosa y Parque de la Cascada en Piriápolis), y la coincidencia de la dirección de esta Formación con la observación de Fragoso César et al. (1987) y Cingolani et al. (1990), de que los sedimentos del Grupo mencionado suelen ocurrir en grabens de dirección NS.

\section{PRINCIPALES ESTRUCTURAS OBSERVADAS Zo-} na Occidental del Ao. Solís Grande Estructuras mayores asociadas a la falla Sarandí del Yi- $\mathrm{A}^{\mathrm{o}}$ - Solís Grande Las milonitas graníticas y las milonitas a granate, grafito y sillimanita se formaron en una faja de cizallamiento E-NE, de edad Proterozoico Inferior (anterior a la intrusión del Granito de Soca, que no aparece deformado por este evento). En el área de este trabajo, la foliación de estas milonitas conforma un arco que evoluciona desde direcciones EW en los alrededores de los Cerros Mosquitos a NS hacia el $\mathrm{A}^{\mathrm{o}}$ Solís Grande, constituyendo un macroindicador cinemático del funcionamiento dextral. Inmediatamente al este del curso de agua antes mencionado, los rumbos de la foliación son NS-N30. El cambio de dirección de foliación arriba señalado pone de manifiesto la existencia de una falla.

Zona Oriental del Ao. Solís Grande Estructuras vinculables a nappes En ocasiones, en las milonitas de Aguas Blancas se han observado pliegues intrafoliales que pueden asociarse a una tectónica de nappes (Fig. 2a). Estas estructuras están retomadas por un evento que genera pliegues abiertos a cerrados de plano axial N20 y eje b 30/N20. La orientación del eje de estos pliegues de segunda generación coincide con la lineación de estiramiento mineral. 

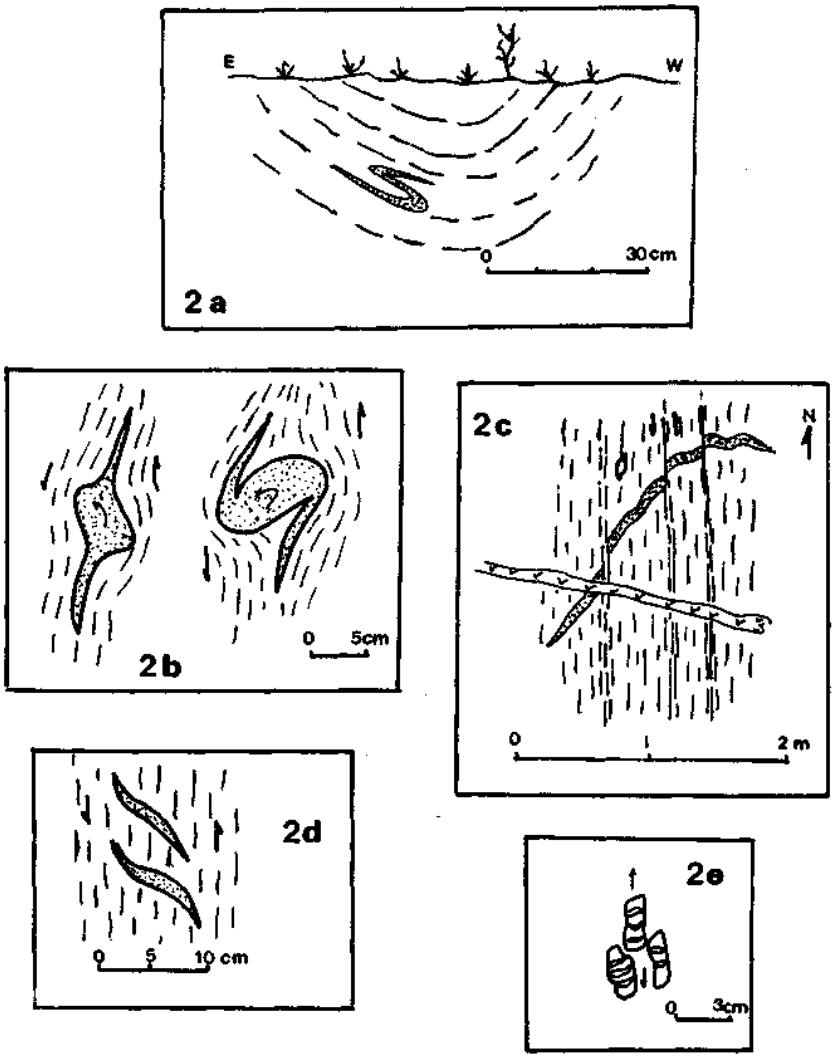

Figura 2 - Estructuras relacionadas a nappes e indicadores cinemáticos de la transcurrencia sinestral Figure 2 - Nappe-related structures and sinistral transcurrency kynematic indicators

Indicadores cinemáticos de la reactivación sinestral de la Falla S.Y.- A.S.G Los indicadores cinemáticos, en afloramientos del Complejo Granítico Solís de Mataojo y de las Milonitas de Aguas Blancas, demuestran una componente sinestral durante la deformación magmática y posconsolidación. (Oyhantçabal et al. 1993).

Los indicadores cinemáticos están representados entre otros elementos estructurales por: sombras de presión y destrucción en megacristales de feldespatos (Fig. 2b), fajas miloníticas de orden centimétrico a hectamétrico, con desplazamiento de filones tardíos (Fig. 2c), y diaclasas en echellon con relleno de cuarzo indicando componentes sinestrales (Fig. 2d). La dirección de extensión NS ha quedado registrada en los cristales de feldespato que presentan fisuras rellenas por epidoto y cuarzo (Fig. 2e).

EVOLUCIÓN GEOLÓGICA Y ESTRUCTURAL Como ya fue mencionado, el área puede ser dividida en dos partes, una al oeste de la falla del $\mathrm{A}^{\mathrm{o}}$ Solís Grande, que forma parte de la gran falla dextral Sarandí del Yí- $\mathrm{A}^{\mathrm{0}}$ Solís Grande, con direcciones estructurales N330 a NS, y la otra al este de este elemento estructural, con direcciones dominantes NS-N30.

Con base en criterios estratigráficos y estructurales se plantea el siguiente esquema evolutivo para dicha zona (Fig. 3 y 4).

1. Cizallamiento E-NE Probablemente durante el Proterozoico Inferior $(2 \mathrm{Ga})$, se produce el cizallamiento que origina las milonitas graníticas y metasedimentarias. A este evento se asocia la Formación Mosquitos e intrusiones graníticas sintectónicas (De Santa Ana et al. 1992).
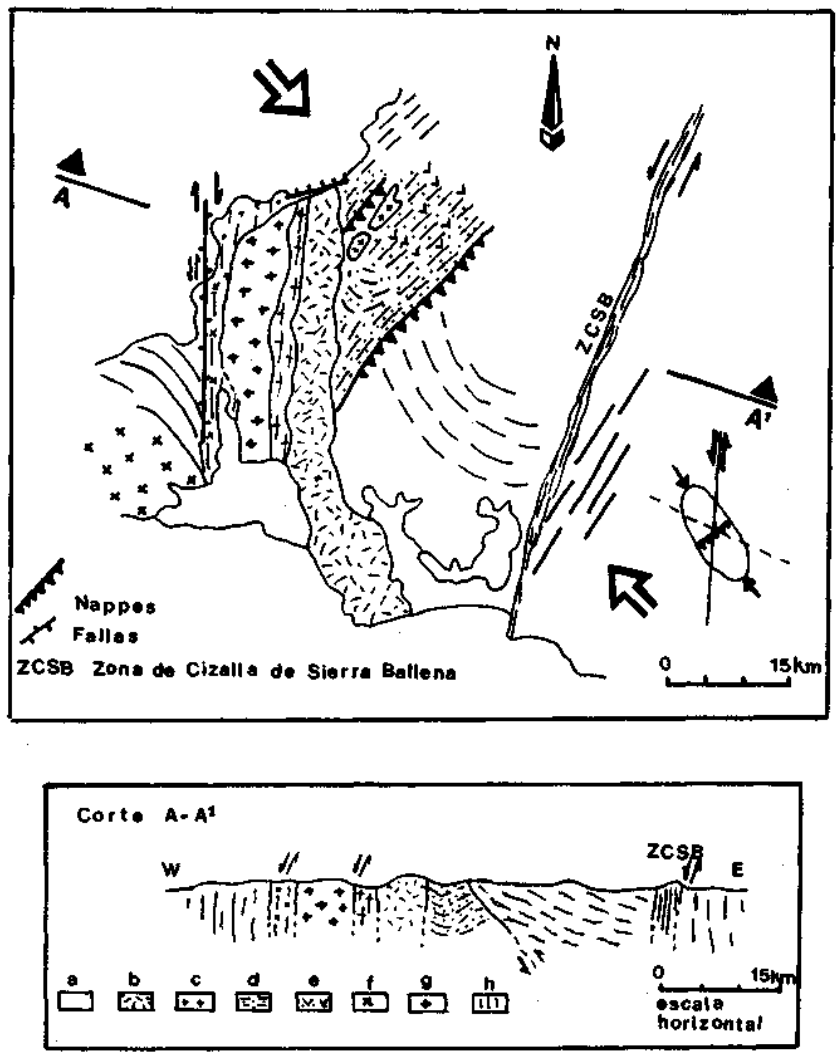

Figura 3 - Posible relación entre las zonas de cizalla y los cabalgamientos. Esbozo en base a Preciozzi et al. (1988) y Fragoso César et al. (1987, 1990). Simbología: a. cobertura sedimentaria; b. Fm. Sierra de Animas; c. granitos post-tectónicos, Grupo Lavalleja; $\boldsymbol{d}$. serie sedimentaria; e. serie vulcano sedimentaria; $\boldsymbol{f}$. granito de Soca; $g$ granitos sintranscurrentes; $\boldsymbol{h}$. basamento con lineaciones estructurales

Figure 3 - Possible relation between shear zones and overthrusting. Sketch based in Preciozzi et al. (1988) and Fragoso César et al. (1987, 1990). Symbols: a. sedimentary cover, b. Sierra de Animas Formation; c. posttectonic granites and Lavalleja Group; d. sedimentary series; e. volcanosedimentary series; f. Soca Granite; g. syn-transcurrent granites; h. basement and structural fabric

2 La falla dextral Sarandí del Yí- $\mathbf{A}^{0}$ Solís Grande Este lincamiento estructural fue reconocido por Preciozzi et al. (1979), con desarrollo entre la localidad de Sarandí del Yí y la Sierra de Animas y dirección N10W. Bossi \& Campal (1992) la denominan Sarandí del Yí Piriapólis y señalan sentido dextral para esa falla. En este trabajo se propone el nombre Sarandí del Yí- A A $^{\circ}$ Solís Grande para este lineamiento, ya que en el sur del país es en ambas márgenes de dicho arroyo donde está bien expuesta la discontinuidad estructural.

Esta gran falla de sentido dextral, que se produjo con posterioridad a la intrusión del enjambre de diques básicos de Florida de edad 1,8 Ga (Rivalenti et al. 1991), produce el arqueamiento de éstos y de las milonitas ubicadas al oeste del $\mathrm{A}^{\mathrm{o}}$ Solís Grande. Este evento sería a su vez anterior a las fases deformacionales de cabalgamiento (nappes) y cizallamiento vertical de la Orogénesis Brasiliana (las edades de rejuvenecimiento de 1450 a $1335 \mathrm{Ma}$ (?) en el Granito de Soca, señaladas por Fragoso César et al. (1987), podrían asociarse a este evento).

Esta falla fue reactivada con sentido sinestral hacia fines del Ciclo Brasiliano, tal como lo demuestran los indicadores 

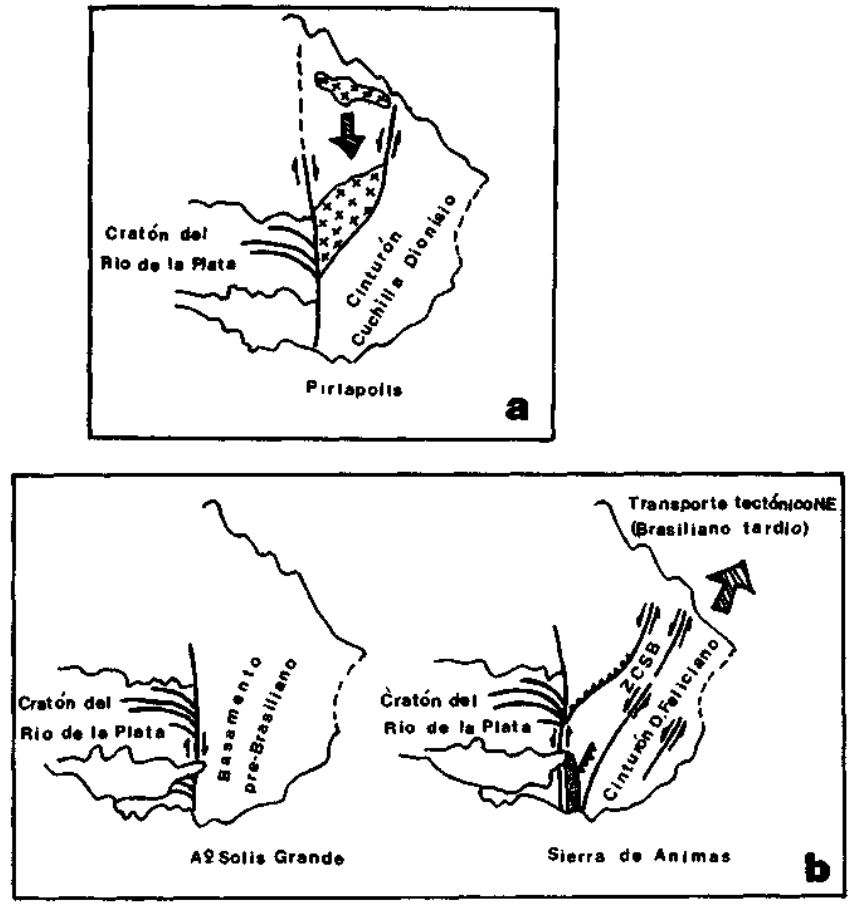

Figura 4-(A) Modelo planteado por Bossi \& Campal (1992). (B) Modelo en base a observaciones en el sur del tinturan y datos de Fragoso Cesar et al. (1990), Masquelin (1990), Bossi \& Campal (1992)

Figure 4 - (A) Model according to Bossi \& Campal (1992). (B) Model based in observations in the southern end of the belt and data by Fragoso Cesar et al. (1990), Masquelin (1990) and Bossi \& Campal (1992)

cinemáticos dentro de la faja milonitizada de la falla. En tanto que la macroestructura constituida por el arqueamiento de los filones y de la foliación milonítica que indican sentido dextral corresponde a un evento anterior. 3. Cabalgamientos vinculados a la etapa colisional temprana del Cinturón Don Feliciano La etapa colisional temprana (sensu Fragoso Cesar et al. 1990), esta- ría representada por los pliegues intrafoliales observados en las Milonitas de Aguas Blancas, en aquellas litologías que corresponderían a escamas del Basamento preBrasiliano.

4. La transcurrencia sinestral y las intrusiones asociadas a la etapa colisional tardía En esta etapa se produce un fuerte cizallamiento que origina fajas miloníticas y determina la intrusión de cuerpos graníticos extremadamente elongados (sin-transcurrentes) en la Faja Móvil. En la zona en estudio se emplaza el Complejo Granítico Solís de Mataojo. Los pliegues abiertos a cerrados, de plano axial N20 y eje b sub-horizontal se asocian a los episodios postumos de esta etapa. Las relaciones entre las transcurrencias y los cabalgamientos se presentan en la figura 3 , donde a su vez se propone que ambas estructuras pueden corresponder a un mismo elipsoide de esfuerzos regional.

5. El reajuste post-levantamiento orogénico y el fallamiento gravitacional asociado Asociado al levantamiento orogénico, se generan grabens de dirección general NS, en los cuales quedan preservados los sedimentos molásicos del Grupo Barriga Negra. En esta zona, el evento está representado por la formación Sierra de Animas y los sedimentos anteriormente citados. Se vincularía a esta etapa la frecuente presencia de filones básicos, de escasa potencia y dirección N320.

CONCLUSIONES La zona en estudio expone en la zona occidental estructuras generadas en el Proterozoico Inferior (Faja de Cizallamiento E-NE), afectadas por la falla dextral Sarandí del Yí - A ${ }^{\circ}$ Solís Grande.

La zona oriental por su parte, expone estructuras generadas durante la evolución del Ciclo Brasiliano: 1. pliegues intrafoliales contenidos en las nappes generadas en la etapa colisional; 2. zonas de cizallamiento transcurrente, intrusiones sin-transcurrentes y pliegues de la etapa colisional tardía; y 3. magmatismo y sedimentación molásica, asociados a los eventos postumos de la evolución del Cinturón. La falla dextral Sarandí del Yi - A ${ }^{\circ}$ Solís Grande es reactivada con sentido sinestral hacia fines del Brasiliano.

Se expresa, especialmente en el área, la importancia que los cizallamientos sub-verticales y las intrusiones sintranscurrentes asociadas tuvieron en la evolución del Cinturón Don Feliciano.

\section{REFERENCIAS BIBLIOGRÁFICAS}

BOSSI, J.; FERNANDEZ, A.; ELIZALDE, G. 1965. Predevoniano del Uruguay. Montevideo, Facultad de Agronomía. (Boletín 78).

BOSSI, J. 1966. Geología del Uruguay. Montevideo, Departamento de Publicaciones, Universidad de la República. $455 \mathrm{p}$.

BOSSI, J. 1989. Recientes Avances Sobre la Geología del Cinturón Orogénico Moderno (1000-500 Ma) en Uruguay. Montevideo, Facultad de Agronomía. (Boletín de Investigación 20).

BOSSI, J. \& CAMPAL, N. 1992. Magmatismo y tectónica trascurrente durante el Paleozoico Inferior en Uruguay. In: GUTIÉRREZ, J. SAAVEDRA, J.; RABANO, I. ed. Paleozoico Inferior en Ibero-América. Extremadura, Universidad de Extremadura, p. 343-356.

CAORSI, J. \& GOÑI, J. 1958. Geología Uruguaya. Montevideo, Instituto Geológico del Uruguay. (Boletín 37).

CINGOLANI, C; SPOTURNO, J.; BONHOME, M. 1990. Resultados geocronológicos y mineralógicos preliminares sobre las unidades Piedras de Afilar y Barriga Negra, R.O.U. In: CONGR. URUG. GEOL., 1. Montevideo, 1990. Actas... Montevideo, SUG. v. 1, p. $11-17$

CORONEL N.; OYHANTCABAL，R; SPOTURNO, J. 1982 Consideraciones estructurales de la Formación Piedras de Afilar en su área tipo, en los alrededores de la estación Piedras de Afilar, Depto. de Canelones, Uruguay. In: CONGR. LATINOAMER. GEOL., 5. Buenos Aires, 1982. Actas... Buenos Aires, AGA. v. 1.

DE SANTA ANA, H.; GOSO, C.; MUZIO, R.; OYHANTCABAL, P. VEROSLAVSKY, G. 1992. Cuenca de Santa Lucía (Uruguay): Evolución tectónica y sedimentaria. In: SIMP. SOBRE BACÍAS CRETÁCICAS BRASILEIRAS, 2. Rio Claro, 1992. Resumos... Rio Claro, UNESP. v. 1, p. 159-161.
FERNANDES, L.; TOMMASI, A.; PORCHER, C. 1992. Deformation patterns in the southern Brazilian branch of the Dom Feliciano Belt: A reappraisal. J/ South Am. Earth Sci., 5(1):75-96.

FRAGOSO CESAR, A.R.S.; WERNICK, E; SOLIANI, E. 1982. Evolução geotectônica do Cinturão Dom Feliciano - Urna contribuição através da aplicação do modelo de Tectônica de Placas. In: CONGR. BRAS. GEOL., 32. Salvador, 1982. Anais... Salvador, SBG. v. 1, p. 13-23.

FRAGOSO CESAR, A.R.S.; MACHADO, R.; GOMEZ RIFAS, C. 1987. Observares sobre o Cinturão Dom Feliciano no Escudo Uruguaio e correlações com o Escudo do Rio Grande do Sul. In: SIMP. SULBRAS. GEOL., 3. Curitiba, 1987. Atas... Curitiba, SBG, v. 2, p. 791809.

FRAGOSO CESAR, A.R.S.; MACHADO, R.; MONTEIRO, R.; SALLET, R. 1990. Nappes e estruturas correlatas do Cinturão Dom Feliciano no Escudo Uruguaio-Sulriograndense: uma introdução ao problema. Acta Geol. Leopold, 13(30):75-92.

JONES, G. 1956. Memoria Explicativa y Mapa Geológico de la Región Oriental del Depto. de Canelones. Montevideo, Instituto Geológico del Uruguay. (Boletín 34).

MASQUELIN, E. 1990. Análisis estructural de las zonas de cizalla de las migmatitas de Punta del Este - Uruguay. Acta Geol. Leopold., 13(30): 137-160

MIDOT, D. 1984. Etude Géologique et Diagnostic Métallogenique pour l'Exploration du Secteur de Minas. París. 175 p. (Tesis de Doctorado, Université Pierre et Marie Curie).

OYHANTCABAL, P.; DERREGIBUS, M.; MUZIO, R.; DE SOUZA, S.; PEEL, E. 1993. Complejo Granítico Solís de Mataojo: evidencias de magmatismo sincolisional relacionado a subducción. In: SIMP. 
INTERN. NEO-PROTEROZOICO CAMBRICO DE LA CUENCA DEL PLATA, 1. Las Palomas, Minas, 1993. Resumenes... Las Palomas, DI.NA.MI.GE. v. 2, trabajo $\mathrm{n}^{\circ} 39$.

PORADA, H. 1979. The Damara - Ribeira Orogen of the Pan-AfricanBrasiliano Cycle in Namibia (south west Africa) and Brazil as interpreted in terms of continental colusión. Tectonophysics, 57:237-265.

PRECIOZZI, E; SPOTURNO, J.; HEINZEN, W. 1979. Carta geoestructural del Uruguay Escala 1:2.000.000. Montevideo, IGU.62 p.

PRECIOZZI, E; SPOTURNO, J.; HEINZEN.W.; ROSSI, P 1988.

Memoria Explicativa de la Carta Geológica del Uruguay a Escala 1/ 500.000 . Montevideo, DINA.MI.GE. $92 \mathrm{p}$.

PRECIOZZI, E; PENA, S.; MASQUELIN, E.; PIAAS, J.; TABO, E 1990. Memoria Explicativa del Fotoplano Piriapolis, Escala 1:100 000. Montevideo, DI.NA.MI.GE/F. Agronomía/F Humanidades y Cien-

cias. $11 \mathrm{p}$.

RIVALENTI, G.; GIRARDI.V.A.; BOSSI, J.; CAMPAL, N.; CIVETTA, L.; MAZZUCHELLI, M.; MOLESINI, M.; LINARI, S.; TEIXEIRA,
W. 1991. Petrology and geochemistry of mafic dike swarms of Uruguay. In: INTERN. SYMP MAFIC DIKES AND RELATED MAGMATISM. Sao Paulo, 1991. Abstracts... São Paulo, SBG. v. 1, p. 41-46.

TOMMASI, A. \& FERNANDES, L. 1990. O Ciclo Brasiliano na porcão sudeste da Plataforma Sul-Americana: um novo modelo In: CONGR. URUG. GEOL., 1. Montevideo, 1990.Actas... Montevideo, SUG. v. 1,p. 107-114.

WALTHER, K. 1927. Contribución al Conocimiento de las Rocas Basálticas Pertenecientes a la Formación Gondwana en América del Sur. Montevideo, Instituto Geológico del Uruguay. (Boletín 13).

MANUSCRITO A779

Recebido em 31 de agosto de 1993 Revisáo do autor em 13 de junho de 1994 Revisáo aceita em 31 de agosto de 1994 